\title{
Role of CT scan in differentiating the type of colorectal cancer
}

\author{
This article was published in the following Dove Press journal: \\ OncoTargets and Therapy \\ 26 April 2017 \\ Number of times this article has been viewed
}

\section{Zhen-Hui Li ${ }^{1, *}$ \\ Ding-Yun You ${ }^{2}, *$ \\ De-Pei Gao' \\ Guang-Jun Yang' \\ Xing-Xiang Dong' \\ Da-Fu Zhang' \\ Ying-Ying Ding'}

'Department of Radiology, The Third Affiliated Hospital of Kunming Medical University, Tumor Hospital of Yunnan Province, Kunming, ${ }^{2}$ Department of Science and Technology, Kunming Medical University, Kunming, Yunnan, People's Republic of China

*These authors contributed equally to this work
Correspondence: Da-Fu Zhang;

Ying-Ying Ding

Department of Radiology, The Third

Affiliated Hospital of Kunming Medical

University, Tumor Hospital of Yunnan

Province, Kunming, Yunnan 650II8,

People's Republic of China

Tel/fax +86 087I68I79594;

+86 087I68I7954I

Email tosney@।26.com;

d_yying@hotmail.com
Objectives: Most colorectal cancers are classical adenocarcinomas (AC), and less frequent subtypes include mucinous adenocarcinomas (MAC) and signet-ring cell carcinomas (SC). The purpose of this study was to evaluate the computed tomography (CT) findings that can help to differentiate MAC and SC from AC.

Methods: $\mathrm{CT}$ scans of 168 patients with pathologically proven MAC and 67 patients with pathologically proven SC were analyzed, and 220 patients with classical AC were also included as a control group. CT findings of the three groups were compared and contrasted in terms of the bowel involvement patterns, contrast enhancement patterns, and presence or absence of bowel obstruction, intratumoral calcification, pericolic fat infiltration, and local tumor extension to adjacent organs. Statistical analyses were made by using the one-way analysis of variance, least significant difference test, and Pearson's chi-square test.

Results: Compared with classical AC, the MAC showed more severe $(6.29 \pm 2.69 \mathrm{~cm}$ vs $4.57 \pm 1.74 \mathrm{~cm}, P<0.001)$ and higher percentage of occurrence of eccentric bowel-wall thickening $(37.2 \%$ vs $11.5 \%, P<0.001)$. Heterogeneous contrast enhancement was most common in MAC $(P<0.01)$, and MAC showed more areas with hypoattenuation $(P<0.001)$. The presence of intratumoral calcification was most frequent in MAC (17.9\% vs $2 \%$ vs $6.8 \%)(P<0.001)$; the $\mathrm{SC}$ also were more severe $(5.75 \pm 2.28 \mathrm{~cm}$ vs $4.57 \pm 1.74 \mathrm{~cm} . P=0.001)$ than $\mathrm{AC}$, but $\mathrm{SC}$ tend to show more cases of concentric even bowel-wall thickening $(67.2 \%)$; homogeneous contrast enhancement was most common in SC $(P<0.01)$, and it showed a target appearance. The presence of peritoneal seeding was most frequent in SC (35.8\% vs $8 \%$ vs $2.7 \%, P<0.001)$, while the presence of regional lymph node metastasis $(P=0.190)$ and direct invasion of adjacent organs or metastasis $(P=0.323)$ were not significantly different among them.

Conclusion: Some radiological features by CT can be used to classify different colon tumor types.

Keywords: cancer, colorectal, mucinous, prognosis, signet ring, computed tomography

Since the World Health Organization introduced the classification of colorectal cancers' histology, the histological subtypes included classical adenocarcinomas (AC), which account for a large majority of cases, mucinous adenocarcinomas (MAC), signet-ring cell carcinomas (SC), and other less frequent forms. ${ }^{1}$ Although computed tomography (CT) has a relatively better prognosis in differentiating $\mathrm{AC}$ and other gastrointestinal malignancies, CT manifestations of other specific histological types of colorectal carcinoma such as MAC and SC are known to be impercipient with a poor prognosis. ${ }^{2,3}$

The MAC and SC are AC in which cancer cells produce excess mucin. A unique pathological feature of SC is the presence of signet-ring cells, which are single 
tumor cells with intracytoplasmic mucin that displaces their nuclei. The infiltrating cells spread diffusely throughout the bowel wall. In contrast, MAC are characterized by abundant extracellular mucin produced by tumor cells. MAC share some clinical characteristics with SC, such as occurring in younger patients ${ }^{4,5}$ and a high propensity for lymph node metastases with low incidence of hepatic metastasis. ${ }^{2,6}$ What is more, its clinical course is aggressive with a high incidence of local recurrence. ${ }^{4-6}$ So the patient's prognosis is usually very poor.

Some case reports regarding the radiological findings of MAC and SC have been reported. ${ }^{7,8}$ At present, despite the fact that CT has been considered as one of the routine workups for preoperative staging in patients with colonic carcinoma, to our knowledge, there have been few reports in the literature describing the $\mathrm{CT}$ features of MAC and SC differing from AC.

The purpose of this study was to retrospectively review the CT features of 455 patients with primary colorectal cancers (AC, MAC, or SC).

\section{Materials and methods}

One hundred seventy-four patients with MAC and 70 patients with SC of the colon and rectum were selected at the Department of Radiology, the Third Affiliated Hospital of Kunming Medical University/Tumor Hospital of Yunnan Province, Kunming, People's Republic of China, during a 7-year period (2008-2015). Two hundred thirty-five patients with $\mathrm{AC}$ of the colon and rectum during a 3-month period were included in this retrospective study. CT was performed in all 479 patients. We excluded 24 patients who showed negative CT findings because of small sizes of the tumors (three cases of MAC, two cases of SC, and 13 cases of AC) and who underwent $\mathrm{CT}$ without enhancement (three cases of $\mathrm{MAC}$, one case of SC, and two cases of AC). Therefore, a total of 168 cases of MAC, 67 cases of SC, and 220 cases of AC constituted the basis of our study. All patients were pathologically confirmed by surgery $(n=402)$ or colonoscopic biopsy $(n=53)$. The study population $(n=455)$ consisted of 257 men and 198 women, and the mean age was 52.5 years with an age range of 15-83 years. The pathological diagnosis of MAC was made according to the criteria that at least $50 \%$ of the tumor was composed of extracellular mucin, typically characterized by pools of extracellular mucin that contains malignant epithelium as acinar structures, strips of cells, or single cells. ${ }^{1}$ When mucin production was intracellular and occurred in $>50 \%$ of neoplastic cells, lesions were histopathologically diagnosed as SC, typically characterized by large mucin vacuoles that fill the cytoplasm and displace the nucleus. ${ }^{1,9}$
CT examination was performed with a 128 -slice CT scanner (SOMATOM Definition AS+, Siemens Medical Solutions, Forchheim, Germany). CT examination included an unenhanced examination with a tube voltage of $120 \mathrm{kVp}$ $(200 \mathrm{~mA} / \mathrm{s}, 0.6$ of pitch, $0.5 \mathrm{~s} /$ rotation, display field of view $42 \mathrm{~cm}^{2}, 512 \times 512$ matrix, and $128 \times 0.6 \mathrm{~mm}$ collimation). Patients were then injected with nonionic contrast material (Ultravist 300, Bayer Schering Pharma AG, Berlin, Germany) with antecubital venous access at a rate of $3.5 \mathrm{~mL} / \mathrm{s}$. A total of $80-100 \mathrm{~mL}$ (1.5 mL per kilogram of body weight) was injected with a CT-compatible power injector (Bracco ACIST EZEM, EmpowerCTA) followed by a $20 \mathrm{~mL}$ saline flush at the same rate. The scanning for contrast enhancement was performed 65-75 seconds after injection.

Retrospective review of CT images was performed in consensus by two radiologists with $>10$-year radiological experience, who were blinded to the pathological results, and a consensus finding was used as the final decision. Site, types of morphological features, length of involved segment, and size of thickness or the mass of involved bowel wall; patterns of bowel-wall thickening (even or uneven, concentric or eccentric); patterns of contrast enhancement (homogeneous or heterogeneous) of the lesion; the degree of pericolic fat infiltration; intratumoral calcification; bowel obstruction; regional lymphadenopathy; and direct invasion of adjacent organs or metastasis were evaluated. During this process, most disagreements in classifying CT features of the lesion were discussed until consensus was reached. For example, if the pattern of bowel-wall thickening of a lesion was classified as concentric pattern by both radiologists, the final decision was concentric pattern. If one radiologist classified the lesion as concentric pattern while another as eccentric pattern, a decision with reader consensus was considered. On a few occasions when consensus cannot be reached by the two radiologists, the third radiologist, who is more experienced, is invited to make the final judgment.

Hyperattenuation of the involved bowel on CT scans was considered present when it enhanced greater than the normal bowel wall, isoattenuated bowel when enhancement was equal, and hypoattenuated bowel when enhancement was low. In cases with the heterogeneous lesion, the extent of hypoattenuated area within the tumor was classified into three groups: $<1 / 3, \geq 1 / 3$ but $<2 / 3$, and $\geq 2 / 3$ of the tumor. Perirectal or pericolic infiltration was graded as grade 1 ( $<1 \mathrm{~cm}$ around the tumor), grade $2(\geq 1 \mathrm{~cm}$ and $<3 \mathrm{~cm}$ ), and grade $3(\geq 3 \mathrm{~cm})^{7,8}$

All statistical analyses were performed by using IBM SPSS statistics Version 19 (SPSS Inc; IBM Corporation Software Group, Somers, NY, USA). Mean values \pm standard 
deviations were given for normal distributed variable. The one-way analysis of variance was used to compare the mean values of all the available continuous variables in the three different groups, and least significant difference test was used for post hoc multiple comparisons. Frequency and percentage were given for categorical data. The chi-square test was used to compare the categorical data in the three different groups. $P<0.05$ was considered statistically significant.

\section{Results}

Comparison of clinical characteristics and CT findings among MAC, SC, and AC is shown in Table 1. The average onset age of SC (43.6 \pm 16.1$)$ was lesser than the MAC

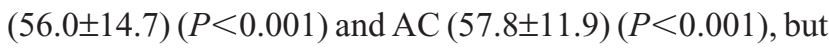

the average onset age of MAC and AC was not significantly different $(P=0.363)$. There was no difference in the sex among MAC, SC, and AC. All tumor types involved the leftsided colon area including the left hemi-colon and rectum most frequently. There was no difference in the involved sites among MAC, SC, and AC. Compared with AC, MAC, and SC involved the right-sided colon to some extent, and the involvement was statistically significant $(P<0.001)$. In morphological types, the annular wall-thickening type was more frequently seen than the mass-forming type in all tumor types $(P=0.006)$. The length of the involved segment in the annular wall-thickening type was similar in both MAC and SC $(P=0.249)$, but longer than AC (MAC vs $\mathrm{AC}, P<0.001$; $\mathrm{SC}$ vs $\mathrm{AC}, P=0.001)$. Compared with

Table I Clinical characteristics and computed tomography findings of patients with MAC, SC, and AC

\begin{tabular}{|c|c|c|c|c|c|c|c|}
\hline Variable & MAC & SC & $A C$ & $P$-value & $P$ (MAC vs SC) & $P$ (MAC vs AC) & $P(S C$ vs $A C)$ \\
\hline Age $\left(\right.$ years) ${ }^{a}$ & $56.0 \pm 14.7$ & $43.6 \pm 16.1$ & $57.8 \pm 11.9$ & 0.000 & 0.000 & 0.363 & 0.000 \\
\hline \multicolumn{8}{|l|}{ Sex } \\
\hline Male & $100(59.5)$ & $36(53.7)$ & $119(54.1)$ & 0.519 & & & \\
\hline Female & $68(40.5)$ & $31(46.3)$ & $101(45.9)$ & & & & \\
\hline \multicolumn{8}{|l|}{ Tumor location ${ }^{\mathrm{b}}$} \\
\hline Right hemi-colon & $54(32.1)$ & $19(28.4)$ & $53(24.1)$ & 0.072 & & & \\
\hline Left hemi-colon & $45(26.8)$ & II (I6.4) & $69(31.4)$ & & & & \\
\hline Rectum & $69(4 I . I)$ & $37(55.2)$ & $98(44.5)$ & & & & \\
\hline \multicolumn{8}{|l|}{ Morphological type ${ }^{b}$} \\
\hline Annular wall thickening & $145(86.3)$ & $67(100)$ & $190(86.4)$ & 0.006 & 0.001 & 0.988 & 0.001 \\
\hline Mass-forming & $23(13.7)$ & 0 & $30(13.6)$ & & & & \\
\hline Length of lesion $(\mathrm{cm})^{\mathrm{a}}$ & $6.29 \pm 2.69$ & $5.75 \pm 2.28$ & $4.57 \pm 1.74$ & 0.000 & 0.249 & 0.000 & 0.001 \\
\hline Thickness of wall $(\mathrm{cm})^{\mathrm{a}}$ & $2.20 \pm 0.92$ & $2.08 \pm 0.73$ & $\mathrm{I} .44 \pm 0.6 \mathrm{I}$ & 0.000 & 0.555 & 0.000 & 0.000 \\
\hline \multicolumn{8}{|l|}{ Pattern of wall thickening ${ }^{c}$} \\
\hline Concentric even & $2(1.4)$ & $45(67.2)$ & $40(2 \mathrm{I} . \mathrm{I})$ & 0.000 & 0.000 & 0.000 & 0.000 \\
\hline Concentric uneven & $89(61.4)$ & $17(25.4)$ & $128(67.4)$ & & & & \\
\hline Eccentric & $54(37.2)$ & $5(7.4)$ & $22(11.5)$ & & & & \\
\hline \multicolumn{8}{|l|}{ Enhancement pattern ${ }^{\mathrm{b}}$} \\
\hline Homogeneous & $7(4.2)$ & $45(67.2)$ & IOI (45.9) & 0.000 & 0.000 & 0.000 & 0.004 \\
\hline Heterogeneous & $161(95.8)$ & $22(32.8)$ & $119(54.1)$ & & & & \\
\hline \multicolumn{8}{|l|}{ Degree of enhancement $\mathrm{t}^{\mathrm{b}}$} \\
\hline Greater & $2(1.2)$ & $38(56.7)$ & $143(65.0)$ & 0.000 & 0.000 & 0.000 & 0.000 \\
\hline Equal & $60(35.7)$ & $18(26.9)$ & $67(30.5)$ & & & & \\
\hline Lesser & $106(63.1)$ & II (I6.4) & $10(4.5)$ & & & & \\
\hline \multicolumn{8}{|l|}{ Extent of low attenuation ${ }^{b}$} \\
\hline$<\mathrm{I} / 3$ of tumor & $33(19.6)$ & $55(82.1)$ & $162(73.6)$ & 0.000 & 0.000 & 0.000 & 0.000 \\
\hline $1 / 3-2 / 3$ of tumor & $44(26.2)$ & $10(14.9)$ & $45(20.5)$ & & & & \\
\hline$>2 / 3$ of tumor & 91 (54.2) & $2(3.0)$ & $13(5.9)$ & & & & \\
\hline \multicolumn{8}{|l|}{ Pericolic fat infiltration ${ }^{b}$} \\
\hline$<\mathrm{l} \mathrm{cm}$ & $|2|(72.0)$ & $26(38.8)$ & I5I (68.6) & 0.000 & 0.000 & 0.542 & 0.000 \\
\hline$>1 \mathrm{~cm}$ & $47(28.0)$ & $4 \mid(6 I .2)$ & $69(31.4)$ & & & & \\
\hline Intratumoral calcification ${ }^{\mathrm{b}}$ & $30(17.9)$ & $2(3.0)$ & $15(6.8)$ & 0.000 & 0.005 & 0.001 & 0.843 \\
\hline Bowel obstruction ${ }^{\mathrm{b}}$ & $10(6.0)$ & $5(7.5)$ & $44(20.0)$ & 0.000 & 0.886 & 0.000 & 0.039 \\
\hline Regional lymphadenopathy ${ }^{\mathrm{b}}$ & $100(59.5)$ & $4 \mid(6 I .2)$ & II $3(5 \mathrm{I} .4)$ & 0.190 & NS & NS & NS \\
\hline Direct invasion or metastasis ${ }^{b}$ & $8(4.7)$ & $6(8.9)$ & $18(8.2)$ & 0.323 & NS & NS & NS \\
\hline Peritoneal seeding ${ }^{\mathrm{b}}$ & $8(4.7)$ & $24(35.8)$ & $6(2.7)$ & 0.000 & 0.000 & 0.429 & 0.000 \\
\hline
\end{tabular}

Notes: a Standard deviations for continuous variables are shown. ${ }^{b} \mathrm{~N}(\%)$ for categorical is shown and the totals for MAC, SC, and AC are I68, 67 , and $220 .{ }^{c} \mathrm{~N}$ (\%) for categorical is shown and the totals for MAC, SC, and AC are 145, 67, and 190.

Abbreviations: AC, adenocarcinomas; MAC, mucinous adenocarcinomas; NS, not significant; SC, signet-ring cell carcinomas. 


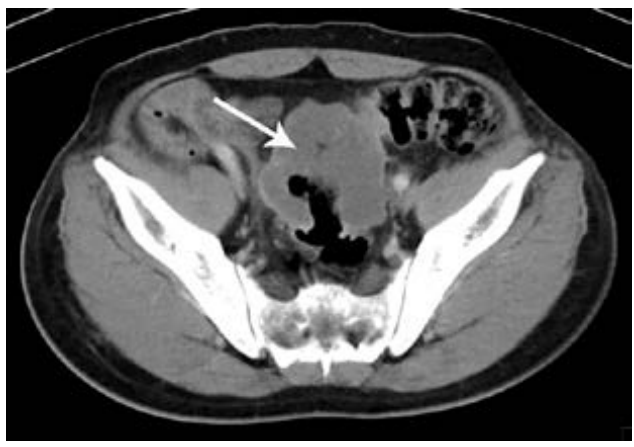

Figure I Computed tomography scan of 52-year-old man with mucinous carcinoma in rectum.

Notes: Computed tomography scan shows severe rectal wall thickening with large areas of low attenuation (arrow).

$\mathrm{AC}$, the MAC and SC were more severe (MAC vs AC, $P<0.001$; SC vs AC, $P<0.001$ ) (Figures 1 and 2), but SC showed more concentric even bowel-wall thickening (Figures 3 and 4), and MAC tended to be more eccentric. Heterogeneous contrast enhancement of the lesion was much more commonly seen in MAC than in $\mathrm{SC}(P<0.001)$ and $\mathrm{AC}$ $(P<0.001)$, and MAC often showed a hypoattenuated area equal to or greater than two thirds of the tumor (Figures 5 and 6). Therefore, the degree of enhancement was less than that of the normal bowel wall in MAC $(P<0.001)$. The SC was inclined to have more homogeneous enhancement, and it showed a target appearance (Figure 7). The presence of intratumoral calcification was more frequently seen in MAC (Figure 8). Peritoneal seeding was more frequent in SC (24/67). Bowel obstruction was more frequently seen in AC (44/220). However, the presence of regional lymph node metastasis $(P=0.190)$ and direct invasion of adjacent organs or metastasis $(P=0.323)$ were not significantly different among them.

\section{Discussion}

Some recent studies have found out that MAC and SC of the rectum show a poor response to neoadjuvant chemoradiation

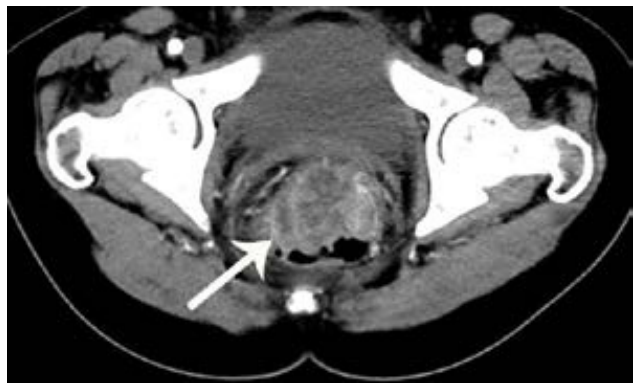

Figure 2 Computed tomography scan of 55-year-old man with nonmucinous carcinoma in rectum.

Notes: Computed tomography scan shows mass-forming rectal wall thickening with heterogeneous enhancement (arrow).

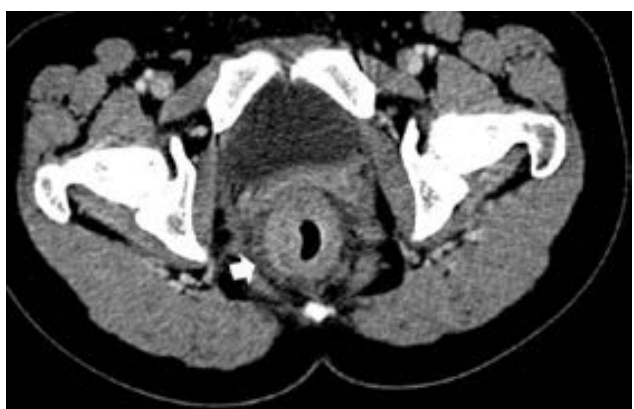

Figure 3 Computed tomography scan of 29-year-old woman with signet-ring cell carcinoma of the rectum.

Notes: Contrast-enhanced computed tomography scan shows concentric rectal wall thickening with target sign. Also noted is diffuse perirectal tumor infiltration (arrowhead). At surgery, tumor had invaded to seminal vesicle.

as seen in terms of larger residual tumors, higher incidence of margin positivity, and greater residual nodal disease. The role of neoadjuvant chemoradiation in MAC and SC of the rectum is of questionable benefit. MAC and SC of the rectum should be treated directly with surgical intervention rather than neoadjuvant chemoradiation. ${ }^{10,11}$ Therefore, histological type of rectal cancer needs to be clarified in preoperative evaluation. Some cases of MAC and SC of the rectum cannot be histopathologically examined because those patients reject colonoscopy. However, MAC and SC may be diagnosed by the CT findings, which is helpful to clinic treatment.

Generally, SC and MAC were considered uncommon histological colorectal cancer subtypes with differences in clinical and histopathological findings. The SC was first reported in the literature by Laufman and Saphir, ${ }^{9}$ and the incidence of SC was reported to range from 0.1 to $2.4 \% .^{9,12-14}$ The incidence of MAC was about $10 \% .^{12,14,15}$ Because of their rare occurrence, the preoperative diagnosis of MAC and SC is difficult. It is important to recognize the specific clinical, pathological, and biological differences among the SC, MAC, and AC. In comparison with $\mathrm{AC}$, both MAC and SC have been shown to be associated

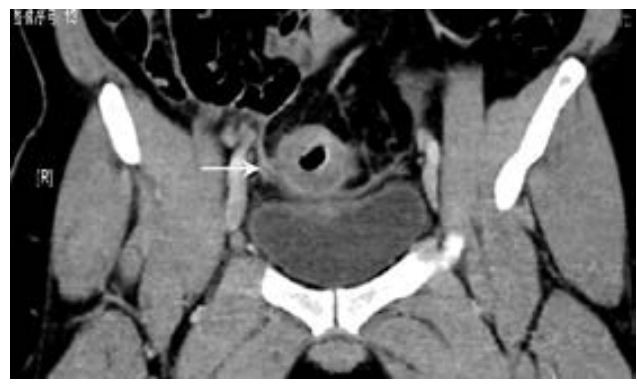

Figure 4 Computed tomography scan of 32-year-old man with signet-ring cell carcinoma of the sigmoideum.

Notes: Contrast-enhanced computed tomography scan shows concentric rectal wall thickening with target sign. At surgery, tumor had invaded peritoneum (arrow). 


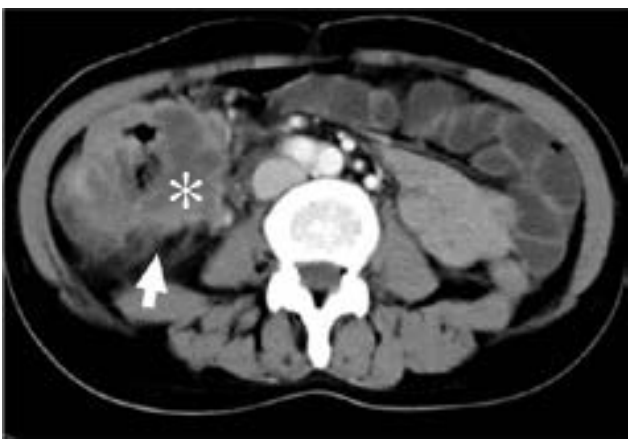

Figure 5 Computed tomography scan of 53-year-old woman with mucinous carcinoma in cecum and proximal ascending colon.

Notes: Computed tomography scan shows concentric uneven bowel-wall thickening along with evidence of pericolic fat infiltration (arrow). Hypoattenuated area is greater than two thirds of tumor (asterisk).

with young age, advanced tumor stage, and accumulation in female patients. Moreover, the tumor spread patterns differ considerably. ${ }^{13,15-17}$ Our study confirmed that the age of $\mathrm{SC}$ at diagnosis was lesser than that of $\mathrm{AC}(P<0.001)$, but the ages of MAC and $\mathrm{AC}$ at diagnosis were not significantly different $(P=0.363)$, and it might be relative to the smaller number of $\mathrm{AC}$ in our study.

Radiologically, there have been a few reports about the CT or MRI findings of MAC and SC. ${ }^{7,8,18,19}$ Kim's study showed that the most common feature of these tumors was a long segment of concentric bowel thickening, and the tumor length was usually $>5 \mathrm{~cm}$ along the longitudinal axis. ${ }^{8}$ In our study, the length of SC was $5.75 \pm 2.28 \mathrm{~cm}$. The thickened wall contrast enhancement showed a target appearance with alternating layers of hyperattenuation in inner and outer zones and middle zone, occurring in $22.4 \%$ (15/67) of our cases. The inner zone was markedly enhanced owing to pathological confirmation of abundant fibrous tissues secondary to desmoplastic response. ${ }^{8}$ Besides, peritoneal seeding of SC $35.8 \%$ $(24 / 67)$ is more significant than MAC $(4.7 \%, P<0.001)$ and

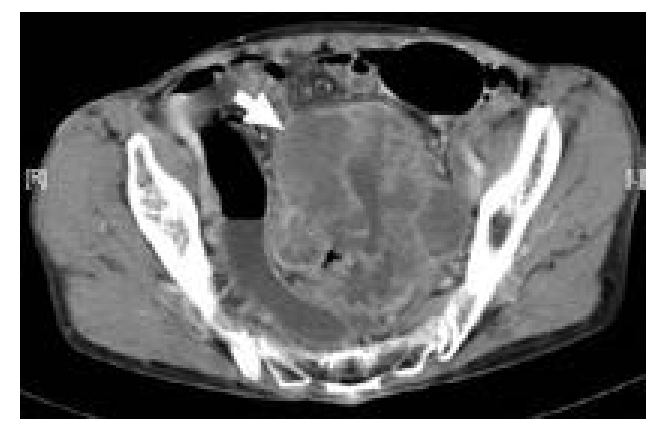

Figure 6 Computed tomography scan of 69-year-old woman with mucinous carcinoma in descending colon.

Notes: Computed tomography scan shows large mass with heterogeneous enhancement. Hypoattenuated area is greater than two thirds of tumor, and enhancement in solid portion of tumor is less than that of normal bowel wall (arrow).

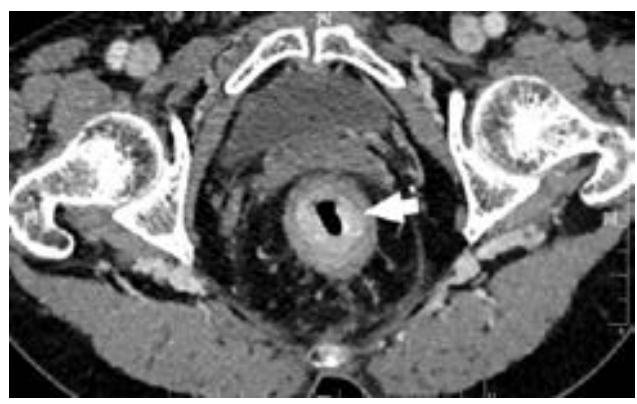

Figure 7 Computed tomography scan of 59-year-old woman with signet-ring cell carcinoma of the rectum.

Notes: Contrast-enhanced computed tomography scan shows concentric rectal wall thickening with target sign (arrow).

AC $(2.7 \%, P<0.001)$ according to our results. This finding is important as it indicates the main pathological process in the mural portion of the bowel wall, and such findings are very rare to be seen in MAC and $\mathrm{AC}$. The SC should be considered in finding these $\mathrm{CT}$ signs such as long segmental bowel-wall thickening, target enhancement, and peritoneal seeding as atypical features of colorectal carcinoma and combined with the age of patient.

In accordance with the majority of published literature reports, ${ }^{7,20}$ MAC showed the presence of CT signs, such as bowel-wall thickness $>2 \mathrm{~cm}$, long segmental and eccentric bowel-wall thickening, heterogeneous enhancement of the lesion with poor enhancement of the solid portion, a large area of hypoattenuation, and intratumoral calcification, which indicated the high likelihood of the MAC. However, in our study, there was no difference in the thickening, involved long segmental bowel wall, and pericolic fat infiltration between MAC and SC ( $P=0.555, P=0.249, P=0.542)$, but MAC and SC appeared more severe than AC $(P<0.01)$. This might be associated with the pathological grading of MAC and SC. Because both MAC and SC were classified

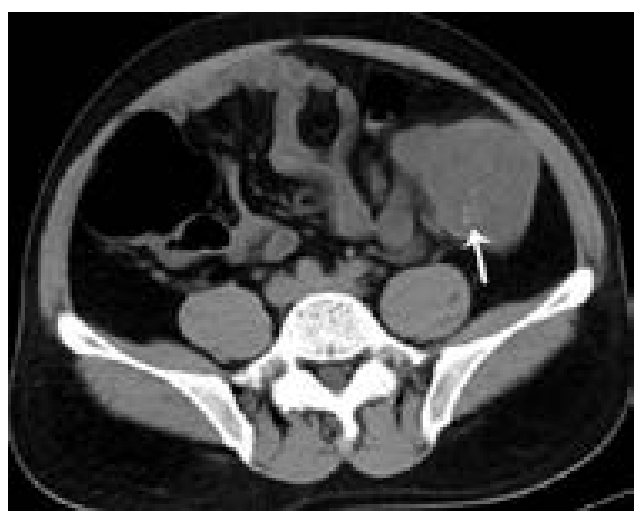

Figure 8 Computed tomography scan of 44 -year-old man with mucinous carcinoma in descending colon.

Notes: Computed tomography scans show small intratumoral calcification (arrow) within hypoattenuated area of eccentric bowel-wall thickening. 
as the poorly differentiated/high grade, ${ }^{21}$ and microsatellite instability occurs more frequently in MAC and SC than in $\mathrm{AC}$ and allows for risk stratification. ${ }^{1,21}$ Therefore, MAC or SC usually showed more aggressive biological behavior than AC. The CT manifestation of MAC showed more uneven concentric and eccentric $(P<0.001)$, more heterogeneous enhancement $(P<0.001)$, and larger hypoattenuated areas (greater than two thirds of the tumor) $(P<0.001)$ than $\mathrm{SC}$ and $\mathrm{AC}$.

Calcification was seen most frequently in MAC, followed by SC and $\mathrm{AC}$, with the incidence being about $17.9 \%$ (30/168), 3.0\% (2/67, $P=0.005)$, and 6.8\% (15/220, $P=0.001)$, respectively. Another interesting $\mathrm{CT}$ finding was bowel obstruction, and it was more frequently seen in AC (20.0\%) than in SC (7.5\%, $P=0.039)$ and MAC $(6.0 \%, P<0.001)$.

The reported atypical morphological features, ${ }^{7}$ such as the broad-based polypoidal lesions, partially polypoid and partially wall-thickening pattern, or a large eccentric mass without significant narrowing of bowel lumen, were more commonly seen in mucinous carcinomas. Our observation was in accordance with them.

\section{Conclusion}

When CT shows a length of concentric bowel-wall thickening and target sign, especially in the rectum or in young patients, $\mathrm{SC}$ should be considered. But when the eccentric bowel-wall thickening is over $2 \mathrm{~cm}$, and intratumoral calcification and heterogeneous enhancement are seen along with a large area of hypoattenuation, especially in the right hemi-colon, there is a high chance of MAC.

\section{Acknowledgments}

This retrospective case series study was approved by the local ethics committee of the Third Hospital of Kunming Medical University, the Affiliated Tumor Hospital of Yunnan Province, the Cancer Hospital of Yunnan Province, Kunming, Xishan Region, People's Republic of China, who waived the need for informed consent to be obtained because this case series is a retrospective study, and patients could not be contacted, as had been discharged from hospital. The medical records and data accessed in the study contained no personal or identifying information. This research was supported by Applied Basic Research Projects of Yunnan Province, China (Grant nos 2014FB062 and 2015FB071).

\section{Disclosure}

The authors report no conflicts of interest in this work.

\section{References}

1. Bosman FT, Carneiro F, Hruban RH, Theise DN. WHO Classification of Tumours of the Digestive System. 4th ed. Geneva, Switzerland: World Health Organization, International Agency for Research on Cancer; 2010.

2. Hosseini S, Zohourinia S, Zare-Bandamiri M, et al. Clinical and pathological characteristics of mucinous colorectal adenocarcinoma: a comparative study. Ann Colorectal Res. 2016;4(1):e34404.

3. Arifi S, Elmesbahi O, Amarti RA. Primary signet ring cell carcinoma of the colon and rectum. Bull Cancer. 2015;102(10):880-888.

4. Tawadros PS, Paquette IM, Hanly AM, Mellgren AF, Rothenberger DA, Madoff RD. Adenocarcinoma of the rectum in patients under age 40 is increasing: impact of signet-ring cell histology. Dis Colon Rectum. 2015; 58(5):474-478.

5. Kanemitsu Y, Kato T, Hirai T, et al. Survival after curative resection for mucinous adenocarcinoma of the colorectum. Dis Colon Rectum. 2003;46(2):160-167.

6. Boustani J, Kim S, Lescut N, et al. Primary linitis plastica of the rectum: focus on magnetic resonance imaging patterns and treatment options. Am J Case Rep. 2014;16(3):581-585.

7. Ko EY, Ha HK, Kim AY, et al. Ct differentiation of mucinous and nonmucinous colorectal carcinoma. AJR Am J Roentgenol. 2007;188(3): 785-791.

8. Kim HJ, Ha HK, Cho KS, et al. CT features of primary colorectal signetring cell carcinoma. J Comput Assist Tomogr. 2001;25(2):225-230.

9. Laufman H, Saphir O. Primary linitis plastica type of carcinoma of the colon. AMA Arch Surg. 1951;62(1):79-91.

10. Charalampakis N, Nogueras González GM, Elimova E, et al. The proportion of signet ring cell component in patients with localized gastric adenocarcinoma correlates with the degree of response to preoperative chemoradiation. Oncology. 2016;90(5):239-247.

11. Jayanand SB, Seshadri RA, Tapkire R. Signet ring cell histology and non-circumferential tumors predict pathological complete response following neoadjuvant chemoradiation in rectal cancers. Int $J$ Colorectal Dis. 2011;26(1):23-27.

12. Kang H, O'connell JB, Maggard MA, Sack J, Ko CY. A 10-year outcomes evaluation of mucinous and signet-ring cell carcinoma of the colon and rectum. Dis Colon Rectum. 2005;48(6):1161-1168.

13. Gopalan V, Smith RA, Ho YH, Lam AK. Signet-ring cell carcinoma of colorectum - current perspectives andmolecular biology. Int J Colorectal Dis. 2011;26(2):127-133.

14. Hyngstrom JR, Hu CY, Xing Y, et al. Clinicopathology and outcomes for mucinous and signet ring colorectal adenocarcinoma: analysis from the national cancer data base. Ann Surg Oncol. 2012;19(9): 2814-2821.

15. Verhulst J, Ferdinande L, Demetter P, Ceelen W. Mucinous subtype as prognostic factor in colorectal cancer: a systematic review and metaanalysis. J Clin Pathol. 2012;65(5):381-388.

16. Kelemen LE, Köbel M. Mucinous carcinomas of the ovary and colorectum: different organ, same dilemma. Lancet Oncol. 2011;12(11): 1071-1080.

17. Chew MH, Yeo SAE, Ng ZP, et al. Critical analysis of mucin and signet ring cell as prognostic factors in an Asian population of 2,764 sporadic colorectal cancers. Int J Colorectal Dis. 2010;25(10):1221-1229.

18. Hussain SM, Outwater EK, Siegelman ES. Mucinous versus nonmucinous rectal carcinomas: differentiation with $\mathrm{mr}$ imaging. Radiology. 1999;213(1):79-85.

19. Deng XC, Zheng B, Tong CY, Jiang S, Zhao TJ. Differentiation between mucinous and nonmucinous colorectal carcinoma based on multi-slice spiral CT findings. Chin J CT MRI. 2015;13(8):80-83.

20. Yan C, Zhu ZG, Yan M, et al. Clinicopathological characteristics and computed tomography features of mucinous gastric carcinoma. $J$ Int Med Res. 2011;39(1):291-301.

21. Aust DE. WHO classification 2010 for the lower gastrointestinal tract: what is new?Pathologe. 2011;32(Suppl 2):S326-S331. 
OncoTargets and Therapy

\section{Publish your work in this journal}

OncoTargets and Therapy is an international, peer-reviewed, open access journal focusing on the pathological basis of all cancers, potential targets for therapy and treatment protocols employed to improve the management of cancer patients. The journal also focuses on the impact of management programs and new therapeutic agents and protocols on

patient perspectives such as quality of life, adherence and satisfaction The manuscript management system is completely online and includes a very quick and fair peer-review system, which is all easy to use. Visit http://www.dovepress.com/testimonials.php to read real quotes from published authors.

Submit your manuscript here: http://www.dovepress.com/oncotargets-and-therapy-journal 